\title{
A lincRNA switch for embryonic stem cell fate
}

\author{
Liuqing Yang ${ }^{1}$, Chunru Lin ${ }^{1}$, Michael G Rosenfeld ${ }^{1,2}$ \\ ${ }^{1}$ Howard Hughes Medical Institute; ${ }^{2}$ Department of Medicine, Division of Endocrinology and Metabolism, University of Califor- \\ nia, San Diego School of Medicine, 9500 Gilman Drive, La Jolla, CA 92093-0648, USA \\ Cell Research (2011) 21:1646-1648. doi:10.1038/cr.2011.166; published online 25 October 2011
}

Cumulative evidences have demonstrated that most of the non-repetitive genome in higher organisms are actively transcribed and surprisingly only a small percentage $(<20 \%)$ of transcripts are associated with genes that encode proteins [1]. One of the emerging themes in the study of non-coding transcripts is large intergenic non-coding RNAs (lincRNAs), a class of large regulatory RNAs implicated in imprinting, dosage compensation, and transcriptional regulation [2]. In light of recent discoveries revealing the flexibility of lincRNAs and their abilities to act as modular scaffolds for protein-chromatin interactions and to form spatially compact arrays of complexes [3, 4], many would acknowledge that most lincRNAs act as sensors and integrators of a wide variety of regulated transcriptional responses and probably epigenetic events, which may have an impact on various human diseases. However, skeptics would suggest that multifocal polymerase entry has no functional significance, and present the challenge of determining whether there is a broad functional network and biological roles of lincRNAs that might regulate gene transcriptional programs involved in homeostasis and human diseases. An initial broad attack on this problem is provided by the report by

Correspondence: Liuqing Yang ${ }^{\mathrm{a}}$, Michael G Rosenfeld ${ }^{\mathrm{b}}$

aE-mail: liy001@ucsd.edu

bE-mail: mrosenfeld@ucsd.edu
Guttman et al. [5], which helps to shed further new light on the "doubts" by delineating a comprehensive network of lincRNAs in a specific cell type and their function as key regulators of global transcriptional programs controlling embryonic stem (ES) cell pluripotency and differentiation (Figure 1). Beyond their importance for our understanding of complexity of non-coding transcripts, these findings show that a previously unsubstantiated network of such influence, as lincRNA can synchronize the activities of different histone-modifying enzymes to regulate gene expression important to the development of life (Figure 1).

The research group, led by Eric Lander at Broad Institute, uncovered a chromatin "signature" for actively transcribed regions between known protein coding genes through a massive sequencing of ChIP data, and used this signature to identify $>3500$ unique forms of lincRNAs in 2009 [6]. Since then, the identification of mechanisms underlying their transcription, regulation, and potential functional roles has been challenging. Studies from several groups suggested a fundamental role of lincRNA in transcription regulation by orientating chromatin-modifying factors/complexes to specific locations in the genome [7, 8]. Now, Guttman et $a l$. use genetic techniques to turn off and on the production of 100 specific lincRNAs in ES cells and find out that one of surprising functions of these transcripts is to coordinate and organize the assembly of chromatin modifying proteins in ES cells, thus permitting the fine tuning of gene expression pattern critical for pluripotent state. Evidence that lincRNAs bind diverse chromatin proteins in ES cells was inferred from an impressive set of computational and experimental data. These included (i) ES cell lincRNAs are globally associated with histone "reading", "writing" and "erasing" complexes; (ii) most of ES lincRNAs are strongly associated with multiple chromatin complexes; (iii) correlation of ES cell gene expression programs with lincRNA-protein interactions (> 40\%). Guttman et al. thus further elucidate two important questions in the field: (i) how many distinct complexes are recruited to various ncRNAs, as reported for individual ncRNAs such as ANRIL [8]? (ii) what is the combinatorial "code" of multiple lincRNA-protein complexes that might be required for maintaining epigenetic memory of ES cells?

Given the widespread involvement of lincRNAs in gene repression and/or activation, acting as sensors of various regulatory signals [9], an immediate question concerns the way in which the functionality of lincRNAs is exerted in cis or in trans. In contrast to what was previously emphasized as a cis acting mode of linRNAs [10], Guttman et al. favor the notion that lincRNAs that they studied based largely on their high levels of expression seem to influence 


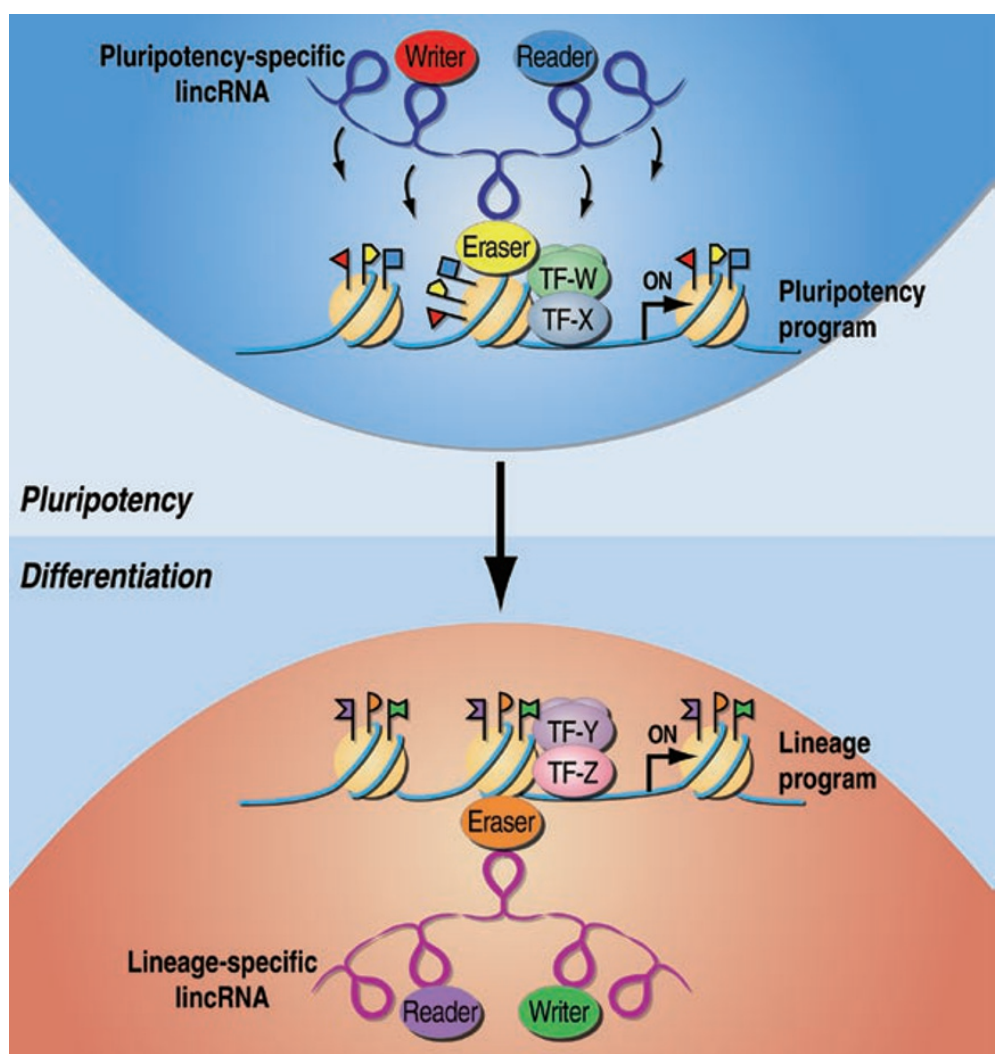

Figure 1 Interplay between cell-type-specific lincRNA-protein complexes determines ES cell transcription programs, which dictates pluripotency vs. differentiation states. Pluripotency- and lineage-specific lincRNAs serve as platforms for assembly of multiple chromatin-modifying complexes, thus licensing the "reading", "writing" and "erasing" of a variety of histone tail marks.

gene expression largely in trans. There are several possibilities for this apparent discrepancy. First, the cis and trans action of lincRNAs might be regulated by signal-induced nuclear architecture changes. Recent evidence suggests that dynamic three-dimensional genomic interactions in the nucleus, in addition to long-range intra- and inter-chromosomal interactions, exert critical roles in regulated gene expression and chromosomal translocations [11]. Cis and trans action of lincRNAs could be switched by signal-induced intra- and inter-chromosomal interactions. It would be intriguing to investigate whether pluripotency and differentiation gene expression in ES cells is associated with nuclear architecture changes and if this is the case, how ES lincRNAs are involved in trans. Second, same lincRNAs may function in cis or in trans depending on their subnuclear locations. It is now evident that the nucleus is a complex, dynamic "organelle" with functional subnuclear domains intimately linked to the genome allowing signaling and ultimately regulation of gene activity [12]. Considering that several noncoding transcripts have been localized to specific subnuclear structures, e.g., nuclear speckles, paraspeckles [13], one might envision that potential relocation of lincRNAs between transcriptionally repressive and permissive environment could switch their action mode.

The findings from Guttman et al. enforce the growing evidence that lincRNAs exert important functional expression of the genome rather than biological noise, suggesting that the genome might encompass intricate lincRNA-based networks that are far more sophisticated than we might have expected. It seems likely that these networks have continued to be harnessed by development in a range of biological processes. From therapeutic perspective, the work of Guttman et al. may make it possible to overcome the challenge of coaxing stem cells into differentiation for therapeutic exploitation. For example, by inhibiting lincRNAs in specific combinations, stem cells could be transformed in specific ways, and this will advance the utility of stem cells for treatment of degenerative diseases.

The report by Guttmann et al. also reminds us that there are many more interesting questions to be answered. First, how do specific proteins interact with lincRNAs and how do these interactions regulate gene transcription? Because of RNA sequence and structural flexibility, it will be of interest to generalize the sequences and the structural motifs of lincRNAs that direct specific protein recognition. Second, it is very interesting that lincRNAs are mostly associated with chromatin-modifying factors. An immediate question is how lincRNA and chromatin-modifying protein interactions promote specialized functions. One possibility is that lincRNAs can allosterically regulate histone "readers", "writers" or "erasers", altering their ability to modulate "repressive" or "activating" epigenetic marks. Third, where are lincRNAs localized within three-dimensional space of nucleus? Analogous to the role of rRNAs in ribosome assembly, lincRNAs can exert functional roles in specific subnuclear organelles and play key roles in regulation of nuclear architecture. Finally, we need to build a catalogue of lincRNAs with common characteristics that will be used to identify and predict the functional features, complemented by experimental analyses in individual cases to determine the mechanisms by which lincRNAs are connected to 
diseases. The development of nextgeneration sequencing and the demonstration of its utility in the identification of non-coding transcripts, intersected with existing molecular techniques from other fields, such as live cell RNA imaging, RNA-protein proteomics and RNA structural biology, suggests that it is likely that many of these questions will be answered in the not too distant future.

\section{Acknowledgments}

We thank J Hightower for artwork. We apologize both to readers and colleagues for references that were omitted owing to editorial/spatial constraints. Work cited from our laboratories was supported by NIH, NCI, DoD and the Prostate Cancer Foundation grants to M G Rosenfeld. Michael G Rosenfeld is a Howard Hughes Medical Institute Investigator. L Yang is the recipient of a DoD Era of Hope Postdoctoral Award (W81XWH-08-1-0554); and C Lin is the recipient of a Susan G Komen for the Cure Fellowship (KG080247).

\section{References}

1 Amaral PP, Dinger ME, Mercer TR, Mattick JS. The eukaryotic genome as an RNA machine. Science 2008; 319:1787-1789.

2 Wang KC, Chang HY. Molecular mechanisms of long noncoding RNAs. Mol Cell 2011; 43:904-914.

3 Tsai MC, Manor O, Wan Y, et al. Long noncoding RNA as modular scaffold of histone modification complexes. Science 2010; 329:689-693.

4 Wilusz JE, Sunwoo H, Spector DL. Long noncoding RNAs: functional surprises from the RNA world. Genes Dev 2009; 23:1494-1504.

5 Guttman M, Donaghey J, Carey BW, et al. lincRNAs act in the circuitry controlling pluripotency and differentiation. Nature 2011; 477:295-300.

6 Guttman M, Amit I, Garber M, et al. Chromatin signature reveals over a thousand highly conserved large noncoding RNAs in mammals. Nature 2009; 458:223-227.

7 Wang KC, Yang YW, Liu B, et al. A long noncoding RNA maintains active chromatin to coordinate homeotic gene expression. Nature 2011; 472:120-124.

8 Yap KL, Li S, Muñoz-Cabello AM, et al. Molecular interplay of the noncoding RNA ANRIL and methylated histone $\mathrm{H} 3$ lysine 27 by polycomb $\mathrm{CBX} 7$ in transcriptional silencing of INK4a. Mol Cell 2010; 38:662-674.

9 Wang X, Song X, Glass CK, Rosenfeld MG. The long arm of long noncoding RNAs: roles as sensors regulating gene transcriptional programs. Cold Spring Harb Perspect Biol 2011; 3:a003756.

10 Ørom UA, Derrien T, Beringer M, et al. Long noncoding RNAs with enhancerlike function in human cells. Cell 2010; 143:46-58.

11 Lin C, Yang L, Tanasa B, et al. Nuclear receptor-induced chromosomal proximity and DNA breaks underlie specific translocations in cancer. Cell 2009; 139:1069-1083.

12 Mao YS, Zhang B, Spector DL. Biogenesis and function of nuclear bodies. Trends Genet 2011; 27:295-306.

13 Bond CS, Fox AH. Paraspeckles: nuclear bodies built on long noncoding RNA. J Cell Biol 2009; 186:637-644. 